\title{
Les gestes de différenciation en éducation physique et sportive : le cas d'un enseignant débutant exerçant en milieu difficile
}

Differentiation in physical education and sports: the case of a novice teacher working in a difficult environment

Teddy Mayeko et Fabienne Brière-Guenoun

\section{OpenEdition} Journals

Édition électronique

URL : http://journals.openedition.org/activites/4474

DOI : $10.4000 /$ activites. 4474

ISSN : $1765-2723$

Éditeur

ARPACT - Association Recherches et Pratiques sur les ACTivités

\section{Référence électronique}

Teddy Mayeko et Fabienne Brière-Guenoun, « Les gestes de différenciation en éducation physique et sportive : le cas d'un enseignant débutant exerçant en milieu difficile ", Activités [En ligne], 16-2 | 2019, mis en ligne le 15 octobre 2019, consulté le 17 octobre 2019. URL : http://journals.openedition.org/ activites/4474; DOI : 10.4000/activites. 4474

Ce document a été généré automatiquement le 17 octobre 2019

\section{cc)}

Activités est mis à disposition selon les termes de la licence Creative Commons Attribution - Pas d'Utilisation Commerciale - Pas de Modification 4.0 International. 


\title{
Les gestes de différenciation en éducation physique et sportive : le cas d'un enseignant débutant exerçant en milieu difficile
}

\author{
Differentiation in physical education and sports: the case of a novice teacher \\ working in a difficult environment
}

Teddy Mayeko et Fabienne Brière-Guenoun

\section{NOTE DE L'ÉDITEUR}

Article soumis le 13 décembre 2018, accepté le 28 juin 2019

\section{Introduction}

\subsection{Enjeux et difficultés de mise en œuvre de la différenciation pédagogique}

Depuis plusieurs décennies, l'ensemble des textes officiels prône le recours à des pratiques différenciées (Legrand, 1986; Meirieu, 1987) afin de placer chaque élève à égalité face aux savoirs, à la culture et aux filières de formation en respectant son rythme et ses modalités d'apprentissage. Légitime et reconnue d'utilité publique, la différenciation fait cependant l'objet de difficultés et de résistances manifestes dans la mise en œuvre des pratiques de classe (Prud'homme, Dolbec, Brodeur, Presseau, \& Martineau, 2005).

2 Dans un rapport du Conseil National d'Évaluation du Système Scolaire (CNESCO) sur la différenciation pédagogique, Chesne, Do, Jego, Briant, Lefresne et Simonis-Sueur (2014) 
signalent que les enseignants sont quotidiennement placés dans des situations professionnelles déstabilisantes les obligeant simultanément à gérer l'avancée du programme, à faire preuve de bienveillance vis-à-vis d'élèves qui n'arrivent pas à suivre et à réaliser leurs objectifs éducatifs dans des contextes d'enseignement difficiles. À cet effet, les recherches actuelles montrent que la mise en place de pratiques effectives de différenciation rencontre plusieurs obstacles liés à la rigidité du cadre pédagogique et didactique de l'enseignant (Crahay, 2003), à l'idéalisation des effets d'un tel processus (Chesne, 2017), à l'individualisation des parcours de formation (Baluteau, 2014 ; Crahay \& Wanlin, 2012 ; Toullec-Théry, 2016) et à la perturbation du climat de travail (Kirouac, 2010). De fait, l'analyse des modalités de différenciation de l'étude révèle que les enseignants se heurtent à un obstacle majeur consistant à individualiser le travail - soit en interagissant avec un élève en particulier, soit en élaborant des dispositifs d'autogestion où chaque élève dispose de ressources pour avancer à son rythme (Crahay \& Wanlin, 2012) - tout en gérant les diverses sollicitations des collectifs de classe (Vors \& Gal-Petitfaux, 2008).

\subsection{Des résistances décuplées en milieu difficile}

3 Ces difficultés se voient renforcées en éducation prioritaire où, en dépit des dispositifs institutionnels spécifiques, le poids du contexte d'enseignement peut limiter les ambitions pédagogiques des enseignants (Ria, 2009) et la poursuite des enjeux de savoirs (Brière \& Poggi, 2015). Les modalités de conduite de l'enseignement qui en résultent vont à l'encontre du projet de réduction des inégalités scolaires que visent les prescriptions. Selon Rochex et Crinon (2011), au-delà des causes exogènes liées aux politiques éducatives, les inégalités scolaires relèvent pour partie de causes endogènes à la classe et rattachées à deux phénomènes qui entraînent un affaiblissement des savoirs étudiés : la restriction curriculaire, qui se caractérise par un abaissement des prescriptions liées aux apprentissages scolaires, et la pédagogie invisible (Rochex, 2016), qui renvoie aux modalités d'enseignement considérées comme innovantes sans toutefois faciliter l'accès aux savoirs des élèves en difficulté.

Dès lors, comprendre les pratiques de différenciation suppose d'analyser les pratiques effectives des enseignants en contexte de classe. À cet égard, les travaux de recherche montrent que les pratiques d'enseignement en milieu difficile se caractérisent par :

a. la mise en place de tâches d'apprentissage souvent moins ambitieuses, décontextualisées et simplifiées (Rayou \& Sensevy, 2014 ; Toullec-Théry \& Marlot, 2013);

b. des contenus de savoir plus flous, moins consistants et fragmentés (Amade-Escot \& Venturini, 2009 ; Kherroubi \& Rochex, 2004);

c. des processus de guidage centrés sur la réussite effective des élèves (dans une dimension pragmatique) et non sur des enjeux épistémiques liés à la conceptualisation des savoirs (Bocchi, 2012).

Aussi la volonté d'établir la "paix scolaire» en conciliant l'installation de la paix sociale et l'adhésion au projet d'enseignement (Butlen, Mangiante-Orsola, \& Masselot, 2017 ; Charles-Pezard, Butlen, \& Masselot, 2012) génère des tensions importantes (Kherroubi \& Rochex, 2004 ; Rouve \& Ria, 2008 ; Saujat, 2010) pouvant limiter la mise en œuvre de stratégies d'enseignement différenciées. Par conséquent, l'analyse des pratiques enseignantes en milieu difficile invite à prendre en compte l'activité réelle des enseignants en situation de travail. Elle révèle en effet la pénibilité de leurs missions (Ria, 2009), liée au coût subjectif que leur demande la mise en œuvre des 
prescriptions en perpétuelle évolution (Bruno, Félix, \& Saujat, 2015) ainsi qu'au manque d'instrumentation et d'outillage dans l'accompagnement des politiques éducatives (Rochex, 2016).

5 En éducation physique et sportive (EPS), les travaux montrent également que la répétition des comportements déviants ${ }^{1}$ oblige les enseignants à résoudre de multiples dilemmes liés à la mise en activité de tous les élèves d'une part et au maintien de la relation didactique d'autre part (Brière-Guenoun, 2015; Gal-Petitfaux \& Saujat, 2018; Monnier \& Amade-Escot, 2009 ; Poggi \& Brière-Guenoun, 2015 ; Vors \& Gal-Petitfaux, 2008). Il leur faut ainsi tout à la fois installer des conditions propices à l'engagement durable des élèves dans le travail (Gal-Petitfaux \& Saujat, 2018 ; Vors \& Gal-Petitfaux, 2008 ; Vors, Gal-Petitfaux, \& Potdevin, 2015), assurer la gestion collective et la gestion individuelle de la classe (Petiot \& Visioli, 2017 ; Vors \& Gal-Petitfaux, 2011) et ajuster en permanence les enjeux de savoir selon les groupes d'élèves et les moments de la leçon (Bière-Guenoun, 2015 ; Poggi, 2014). À titre d'exemple, dans une approche dérivée du cadre de l'anthropologie cognitive, Vors et Gal-Petitfaux (2009) soulignent que certaines stratégies dites de masquage ou d'ostension sont destinées à préserver la dynamique collective de travail et la viabilité de la relation pédagogique. Ces auteurs mettent aussi en évidence des formes d'organisation triadique de l'activité individuelle des élèves en lien avec trois préoccupations typiques: travailler les exercices demandés, s'amuser à se défier entre pairs, vagabonder en quête d'occupations (Vors \& Gal-Petitfaux, 2011). En milieu difficile, l'enchâssement de ces trois faisceaux de préoccupations apparaît de manière cyclique au cours de la leçon et se caractérise par une occupation singulière de l'espace. En somme, il apparaît que la perception de ces différentes formes d'activité, comprenant nécessairement des déviances furtives, peut permettre aux enseignants de considérer les situations comme moins instables et imprévisibles (Monfroy, 2002 ; Vors \& Gal-Petitfaux, 2011).

\subsection{Un renforcement des difficultés chez les enseignants débutants}

6 De façon similaire, les recherches actuelles mettent en évidence le caractère composite, situé et conflictuel de l'activité des enseignants débutants, fréquemment nommés en éducation prioritaire ${ }^{2}$.

7 Ces derniers éprouvent plus de difficultés que les enseignants chevronnés à instaurer une relation pédagogique stable, à créer les conditions de la réussite en classe et à contrôler le déroulement de la séance (Gal-Petitfaux, Vors, \& Cizeron, 2011 ; Ria, 2009 ; Ria, Saury, Sève, \& Durand, 2001). Dans une recherche centrée sur les pratiques des enseignants stagiaires exerçant en éducation prioritaire, Butlen et Masselot (2018) montrent, selon une approche didactique, que les débutants peinent à installer la « paix scolaire", à exercer une vigilance didactique et à gérer le couple de processus dévolution/institutionnalisation (p. 64). Les travaux actuels soulignent également que les ressources dont disposent les enseignants s'enracinent dans les prescriptions officielles, mais aussi dans le dépassement des tensions et des dilemmes qu'ils vivent lors de leurs premières expériences en classe (Brière-Guenoun, 2017 ; Saujat, 2010). Chaque jour, des néophytes se trouvent ainsi confrontés à des situations pénibles, pouvant les amener à faire «le deuil des exigences scolaires ou d'une conception idéalisée de leur métier» (Ria, 2009). En conséquence, ils abandonnent plus vite les 
objectifs de différenciation pour se recentrer sur le maintien de l'ordre, qui apparaît comme un aspect central de leurs préoccupations.

En EPS, certains auteurs montrent par exemple que les enseignants débutants construisent des seuils de tolérance plus flous vis-à-vis des comportements déviants, sont moins capables d'alterner les temps forts et les temps faibles d'apprentissage et interviennent assez peu sur les contenus (Vors \& Gal-Petitfaux, 2008). Par ailleurs, ils ont du mal à déceler les indices pertinents de l'activité d'apprentissage des élèves (Brière-Guenoun, 2017). Ces divers éléments expliquent, en partie, pourquoi les enseignants débutants sont globalement en difficulté pour accompagner et différencier l'apprentissage des élèves en classe.

9 Ainsi, dans le prolongement de ces travaux, nous souhaitons étudier les modalités de différenciation lors de pratiques effectives en EPS, considérant qu'elles recèlent un véritable enjeu de lutte contre les inégalités d'accès aux savoirs, notamment en milieu difficile. Nous tenterons donc de comprendre comment un enseignant d'EPS débutant exerçant en éducation prioritaire concilie les diverses contraintes qui pèsent sur son activité, liées aux caractéristiques du contexte, à ses motifs d'agir et aux prescriptions institutionnelles pour conduire et différencier son enseignement.

\section{Cadre théorique}

\subsection{Un double ancrage pour caractériser l'activité de l'enseignant}

10 Notre ancrage théorique repose sur une approche comparatiste, prenant appui sur le modèle de l'action conjointe en didactique (Sensevy, \& Mercier, 2007), auquel sont incorporés des fondements de l'ergonomie tels que développés en clinique de l'activité $(\text { Clot, 1999) })^{3}$.

11 Le premier courant de recherche s'intéresse à la dynamique des interactions didactiques produites en classe afin de mettre en évidence la co-construction des objets de savoir au cours des situations d'enseignement (Mercier, Schubauer-Leoni, \& Sensevy, 2002). Il a pour but d'examiner les façons dont l'enseignant et les élèves contribuent respectivement (ou non) à faire avancer le temps didactique. De fait, le travail de l'enseignant se décline en divers gestes dépendants de l'activité des élèves, des prescriptions officielles et des pratiques culturelles (Brière-Guenoun, 2017). Les modalités interactives de construction des savoirs confèrent aux gestes de l'enseignant une relative singularité, mais reflètent également l'appartenance à un genre professionnel (Clot, 1999).

12 Selon les orientations développées par l'approche clinique, l'activité réalisée ne se confond pas avec le réel de l'activité, mais intègre également des possibles non réalisés, conceptualisés par Clot (1999) en tant qu'activité contrariée, suspendue ou empêchée. Ainsi, à travers les méthodologies d'entretiens indirectes qu'elle développe (autoconfrontations simples et croisées notamment), cette orientation scientifique permet d'analyser l'activité du sujet dans ses dimensions objectives et subjectives. Elle vise à révéler certaines tensions vécues par les enseignants entre le respect des prescriptions institutionnelles (ce que les enseignants doivent faire), leurs intentions didactiques (ce que les enseignants veulent faire) et le déroulement de l'activité effective (ce que les enseignants font). Au-delà, elle met en évidence le style de l'enseignant et les manières dont il se nourrit du genre du métier. 


\subsection{L'étude du réel de l'activité de l'enseignant : entre dimensions didactiques et registres de métier}

13 S'intéresser au réel de l'activité de l'enseignant nécessite d'investiguer les dimensions didactiques de son travail, elles-mêmes interrogées à partir des orientations théoriques développées en clinique de l'activité.

14 Afin de comprendre comment l'enseignant conduit l'étude, nous empruntons au modèle de l'action conjointe en didactique les descripteurs de l'action du professeur (Sensevy, \& Mercier, 2007) qui permettent de décrire les manières dont il :

a. définit les tâches mises à l'étude, qui concernent les règles visant à poser les objets, les enjeux et les conditions de l'apprentissage ;

b. dévolue aux élèves la responsabilité de se mettre au travail en s'emparant des contenus d'enseignement et des conditions de la tâche ;

c. régule l'activité des élèves afin de les amener à progresser au cours de l'étude ;

d. institutionnalise les savoirs et les pratiques légitimes afin de construire des références communes et partagées au sein de la classe.

Ces actions didactiques, emblématiques de l'activité de l'enseignant sont destinées à organiser les apprentissages dans l'interaction avec les élèves. En cela, elles contribuent à redéfinir les places respectives de l'enseignant et des élèves (topogenèse) et assurent l'avancée des savoirs dans le temps (chronogenèse) grâce à la construction du milieu didactique dont l'enjeu est de favoriser l'élaboration d'une référence commune et partagée au sein de la classe (mésogenèse).

15 Pour prolonger notre analyse sur l'activité de l'enseignant et comprendre ce qui se joue au-delà de la situation présente, il s'agit de mettre en évidence les conflits de préoccupations qui sous-tendent les manières dont il vit son métier, voire se transforme dans le métier (Clot, 2008). D'après Kostulski, Clot, Litim et Plateau (2011, p.134), «le métier peut être défini comme une architecture multidimensionnelle articulant quatre instances en tension». Il associe les instances impersonnelle (dimension prescriptive et conceptuelle du travail), interpersonnelle (dimension collective de l'activité), transpersonnelle (dimension sociale, historique et culturelle de la profession) et personnelle (dimension singulière qui fait écho au style propre de l'enseignant). Les manières dont l'enseignant s'approprie ces registres du métier renvoient aux déterminants (ou déterminations) de l'action didactique qui recèlent les potentialités de développement ou d'empêchement de son activité (BrièreGuenoun, 2017).

16 Ainsi, son activité s'inscrit au carrefour de multiples influences d'ordre contextuel, interpersonnel, subjectif, épistémique, institutionnel et culturel (ibid.). Dans notre recherche, nous interrogerons les manières dont l'enseignant conduit et différencie l'étude des élèves en pointant l'influence de ces différents éléments sur son activité.

\subsection{L'analyse des modalités de différenciation de l'enseignant}

17 Eu égard aux prescrits institutionnels, le travail de l'enseignant consiste à conduire et à organiser l'étude afin d'amener tous les élèves à construire, en classe, des connaissances et des compétences plus élaborées. Cependant, la diversité du public scolaire - se traduisant entre autres par des dispositions cognitives et motrices 
plurielles - induit des remaniements importants qui pèsent sur l'activité de l'enseignant et contribuent à une redéfinition des normes contractuelles. Selon Schubauer-Leoni (1996), les relations entre l'enseignant et les élèves sont pour partie régies par un contrat didactique qui peut s'appliquer différentiellement en fonction de la position scolaire occupée : les élèves ne sont pas tout à fait sollicités de la même manière par l'enseignant s'ils sont a priori considérés comme "forts " ou "faibles " (Schubauer-Leoni, 1986). Ce mécanisme peut relever d'une origine implicite ou inconsciente (Rochex, 2016), et est étroitement lié aux représentations des enseignants qui commandent des logiques de fonctionnement différenciatrices. Sur ce point, Kherroubi et Rochex (2004) montrent qu'en éducation prioritaire les représentations que les enseignants développent à propos des possibilités de leurs élèves les conduisent fréquemment à abaisser, de façon injuste et trop marquée, les exigences vis-à-vis du savoir. En outre, cette différence de sollicitation traduit également une volonté d'ajuster les contenus de savoir aux possibilités réelles des élèves (Schubauer-Leoni, \& Leutenegger, 2002) et/ou de suivre les prescriptions institutionnelles qui insistent largement sur la mise en œuvre d'une pédagogie différenciée. Ici l'enjeu n'est pas de créer une différenciation des apprentissages (qui existe déjà naturellement) «mais d'en contrôler le processus » (Meirieu, 2013) en aménageant l'étude selon les dispositions des apprenants. Par conséquent, le contrat didactique différentiel (Schubauer-Leoni, 1996) est un outil pertinent pour étudier la dynamique contractuelle qui s'instaure entre l'enseignant et les différents élèves de la classe à propos des enjeux de savoir.

Ainsi, afin d'analyser les modalités de conduite et de différenciation de l'étude, nous cherchons à caractériser l'activité de l'enseignant conjointement à l'activité des élèves telle qu'elle se manifeste dans l'interaction didactique tout en mettant en évidence les conflits de préoccupations qui l'animent. Deux questions principales ont ainsi guidé notre recherche :

- Quels procédés l'enseignant utilise-t-il pour conduire l'étude dans la classe et différencier son enseignement en fonction des profils des élèves?

- Quelles sont les préoccupations au fondement de son activité et en quoi renvoient-elles à des dilemmes du métier?

\section{Méthodologie}

\subsection{Le contexte de l'étude}

19 Nous avons observé les quatre premières leçons (sur huit) d'une séquence de tennis de table avec un enseignant débutant ${ }^{4}$ (partiellement spécialiste de l'activité), nommé David, dans une classe de quatrième (composée de 23 élèves) en éducation prioritaire ${ }^{5}$. $\mathrm{Eu}$ égard à nos remarques antérieures, les élèves de cette classe montraient peu d'intérêt pour les tâches scolaires et possédaient des caractéristiques typiques de l'établissement et plus généralement des milieux difficiles. De plus, n'ayant encore jamais vécu une séquence d'enseignement dans cette activité physique sportive et artistique (APSA) ils visaient l'acquisition de la compétence attendue de niveau 1 des programmes de collège ${ }^{6}$. Cette dernière précise que les élèves doivent « rechercher le gain loyal d'une rencontre en assurant la continuité de l'échange, en coup droit ou en revers et en profitant d'une situation favorable pour le rompre par une balle placée latéralement ou accélérée (...) ». 


\subsection{Le dispositif de recueil des données}

Le dispositif méthodologique s'inspire de celui proposé par Schubauer-Leoni et Leutenegger (2002) et des remaniements opérés pour intégrer la dimension clinique de l'activité (Brière-Guenoun, 2017). Il se compose de trois phases distinctes :

- un entretien ante-cycle de type semi-directif destiné à faire émerger les intentions préalables de l'enseignant, et notamment ses choix d'enseignement relatifs à la structuration des leçons et aux objets d'enseignement abordés ;

- des séquences vidéographiques portant sur les moments clés du synopsis de leçon qui ciblent les moments saillants de l'intervention de l'enseignant (en référence aux quatre actions didactiques) et leurs incidences sur l'activité d'apprentissage des élèves ;

- des entretiens post-séances, de type «autoconfrontation» (EAC) menés selon les orientations de la clinique de l'activité (Clot, 1999) permettant à l'enseignant de revivre et d'analyser son activité à partir du visionnage des enregistrements filmés et visant à mettre au jour les conflits de préoccupations sous-jacents à son intervention in situ. Ces entretiens d'une durée d'environ $1 \mathrm{~h} 15$ ont été enregistrés de façon audio et ont été pilotés par son choix d'arrêter la vidéo. Ils ont donc été menés en partant des commentaires initiés par l'enseignant tout en étant prolongés par des relances centrées sur les questions de recherche lorsque les propos de l'acteur le permettaient.

\subsection{Le traitement des données}

21

Le traitement des données consiste à étudier le travail de l'enseignant selon une double dimension temporelle (Ligozat, 2008) croisant une échelle mésodidactique (les leçons ou la séquence) et une échelle microdidactique (des évènements remarquables liés à des tâches spécifiques).

La première étape, réalisée à l'échelle mésodidactique, avait pour but d'établir le synopsis de chacune des leçons en identifiant les types de tâches mis en place et les enjeux de savoirs associés selon le niveau des élèves. Le travail d'analyse fut permis grâce à la construction de tableaux ou de graphes, représentatifs de l'activité prévue par l'enseignant pour agencer le temps et l'espace.

Puis dans un second temps, nous avons extrait des "évènements remarquables " (Leutenegger, 2009), considérés comme des éléments saillants nous permettant d'accéder aux processus de conduite et de différenciation des apprentissages mises en œuvre par l'enseignant. Ces évènements ont été choisis en raison de modalités spécifiques de différenciation selon la position scolaire des élèves («forts» ou «faibles»). Par exemple, lorsque l'enseignant aménage une tâche ou transmet une consigne à destination exclusive d'un élève ou d'un groupe d'élèves. Leur traitement repose sur le croisement d'analyses a priori et a posteriori. La première (analyse a priori) définit les "contraintes et les possibles de la tâche observée" (Leutenegger, 2000) et représente un discutant des fonctionnalités didactiques de la tâche. La seconde (analyse a posteriori) se focalise sur les actions spécifiques de l'enseignant (définition, dévolution, régulation, institutionnalisation) référées aux conduites motrices des élèves, identifiées selon plusieurs niveaux, afin d'apprécier les manières dont il conduit et différencie l'étude. Ces évènements sont ensuite requestionnés dans les entretiens post, sous forme d'autoconfrontation (EAC), dans l'optique de comprendre les conflits de préoccupations 
caractérisant le réel du travail de l'enseignant. Précisons également que pour chacune de ces deux étapes, les données ont été mises en relation avec l'entretien ante qui précise les intentions préalables de l'enseignant inhérentes aux objectifs et aux modalités de travail.

\section{Résultats}

Dans cette section, nous présenterons succinctement l'analyse mésodidactique qui met en évidence l'actualisation de l'avancée du temps didactique dans les dispositifs et les objets de savoirs ainsi que les formats pédagogiques et les organisations communicationnelles en fonction du profil des élèves. Puis nous reviendrons sur deux évènements remarquables qui traduisent les modalités différentielles de conduite de l'enseignement se rapportant respectivement au choix des objets matériels et aux formats de communication.

\subsection{Analyse mésodidactique : l'agencement des dispositifs et l'organisation de l'étude au service de la différenciation des enjeux de savoirs}

Conformément aux recommandations des programmes, la séquence de tennis de table s'organise autour de la notion d'affrontement. Les élèves doivent apprendre à identifier une situation favorable de marque pour ensuite saisir des opportunités d'action et rompre l'échange par une balle placée ou accélérée. Ces prescrits institutionnels, renvoient à deux enjeux de savoir distincts : le jeu accéléré et le jeu placé. Ils pilotent ainsi la construction de la séquence et induisent de la part de l'enseignant des adaptations particulières en fonction du niveau de pratique des élèves.

En premier lieu, l'analyse mésodidactique des dispositifs mis à l'étude au cours de la séquence d'enseignement (Tableau 1) montre que David propose des tâches différenciées en fonction du profil des élèves. 
Tableau 1 : Synopsis des quatre premières leçons. Tableau 1: Synopsis of the first four lessons

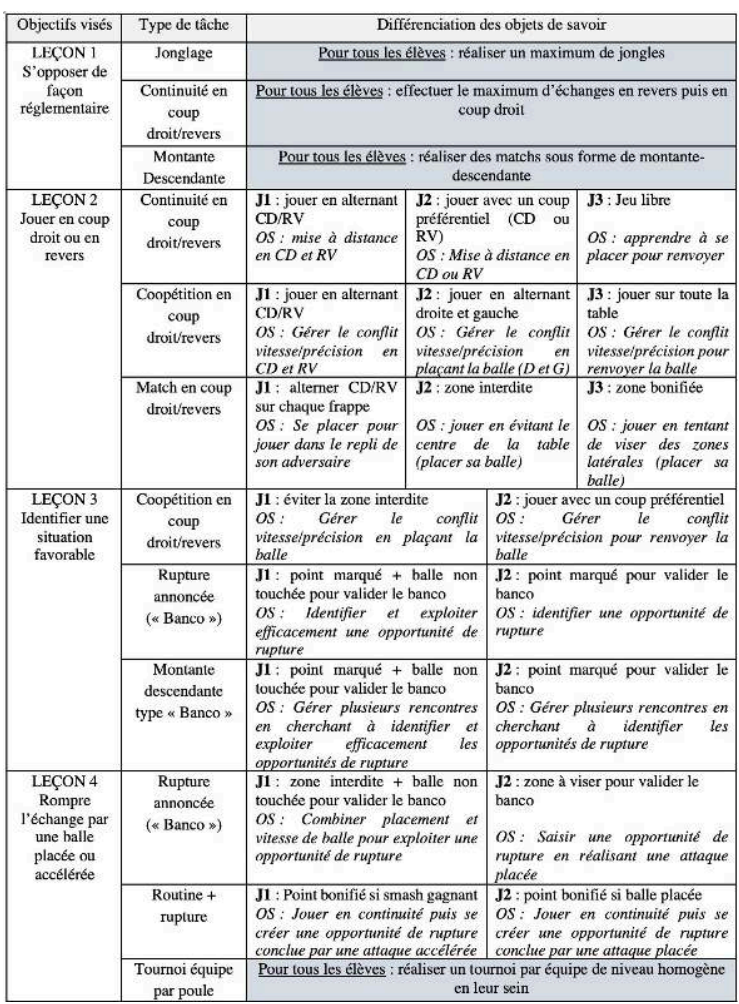

Légende du tableau 1

$\mathbf{J 1}$ : joueurs de niveau 1 (plus près des exigences programmatiques)

$\mathbf{J 2}$ et $\mathbf{J 3}$ : joueurs de niveaux 2 et 3 (plus éloignés des exigences programmatiques)

os : Objet de savoir visé par les élèves au sein de la tâche

CD/RV : Coup droit/revers

En effet, on constate une différenciation constante des tâches et des objets de savoir entre la deuxième et la quatrième leçon. L'enseignant oriente les élèves les plus éloignés des attendus de la compétence vers le jeu en placement (dans le Tableau 1, voir J2 et J3) et les autres élèves, plus proches des exigences des programmes, vers le jeu en vitesse (dans le Tableau 1 voir J1). Cette lecture dichotomique de la compétence attendue le conduit donc à proposer aux élèves des tâches différentes révélant sa propre reconfiguration des prescriptions et ses buts subjectifs. Selon ce découpage, les élèves en difficulté doivent apprendre en priorité à renvoyer la balle réglementairement et à saisir des opportunités de rupture par des balles placées tandis que les élèves en réussite doivent être capables de se créer des opportunités de rupture et de les exploiter par des balles placées et accélérées. À ce titre, l'introduction de différents savoirs centrés sur l'apprentissage de la rupture de l'échange est corrélée à un travail par atelier permettant à David de différencier les objets d'acquisition en fonction des compétences technico-tactiques des élèves. Alors que les dispositifs proposés aux élèves "forts" (capables de produire des frappes sécuritaires et en contrôle) ont pour objectif l'acquisition de contenus relatifs à la posture et à la linéarité des trajectoires de balle, ceux proposés aux élèves «faibles » sont centrés sur la tenue de raquette et l'orientation du tamis. L'agencement des dispositifs dans le temps fait écho aux intentions didactiques différentielles de l'enseignant et à ses représentations concernant le niveau des élèves : "Les meilleurs, je les oriente vers le jeu en vitesse tandis que les moins bons doivent d'abord apprendre à placer la balle» (entretien post séance 3). 
Dans cet extrait d'entretien, David légitime ses choix au regard d'une analyse préalable (ou évaluation diagnostique) et de la compétence attendue. La formulation de la compétence l'incite explicitement à travailler sur deux modalités de rupture au cours de la séquence : le placement et l'accélération. Dès lors, il semble que l'activité de David est contrainte par un prescrit institutionnel relativement étroit (la compétence attendue), influençant probablement son analyse concernant l'activité des élèves. En établissant deux catégories distinctes d'élèves (les "forts » et les " faibles »), à la suite de l'évaluation diagnostique réalisée en leçon 1, David peut proposer des tâches et des savoirs différenciés en fonction des besoins estimés de chaque profil (voir Tableau 1).

D'autre part, l'organisation de l'étude, et en particulier le choix des formats pédagogiques et communicationnels (voir le Tableau 2), soutient les intentions différentielles de l'enseignant tout en répondant à des préoccupations de gestion de classe. En effet, à diverses reprises David réorganise les dispositifs d'étude, en jouant notamment sur les dimensions spatiales et matérielles, pour prendre en charge les comportements transgressifs des élèves et ainsi restaurer un climat de travail plus stable: "Quand les élèves se mettent à ne plus vraiment travailler, il faut vite intervenir, car ils n'apprennent pas et peuvent vite gêner les autres" (entretien ante). En ce sens, la mésogenèse s'enrichit de ces aménagements qui permettent à l'enseignant de prendre en compte les attitudes de déviances tout en respectant les différences de niveaux entre les élèves. Autrement dit, l'organisation des tâches sur les différentes leçons montre que la réactualisation des groupes et des espaces de travail (et plus spécifiquement le déplacement des tables à tel ou tel endroit) est une ressource pédagogique permettant à l'enseignant de redéfinir certaines habitudes dans une optique d'apprentissage et de gestion de groupe.

Tableau 2 : Formats pédagogiques et structures de communication au fil des quatre leçons. Tableau 2: Educational formats and communication structures during the first four lessons

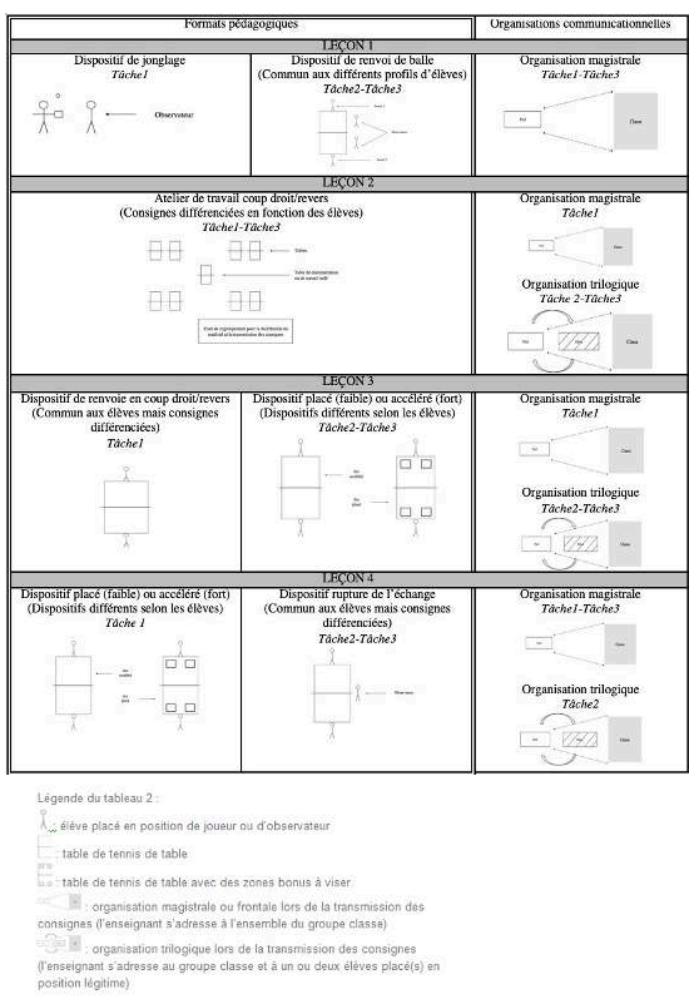


29 Le tableau 2 a été construit a posteriori de nos observations en effectuant un recoupement entre le synopsis des leçons, les formats pédagogiques mis en œuvre et les structures de communication employées. Son analyse montre que David mobilise deux formats de communication pour définir les tâches. Le premier est une organisation dilogique (Leutenegger, 2008) ou magistrale qui consiste à transmettre une consigne à l'ensemble des élèves de la classe afin de les orienter vers un objet de savoir clairement défini. Le deuxième est une organisation trilogique (Leutenegger, 2008) permettant d'assurer des échanges privilégiés entre trois instances : l'enseignant, un élève placé en position légitime et le reste des élèves de la classe, alors considérés comme une entité collective. Contrairement à l'organisation magistrale, ce type de format permet à l'enseignant d'exposer clairement le but de la tâche et de présenter les différents aménagements qui président à l'organisation du travail. En ce sens, la mésogénèse progresse grâce aux formats de communications trilogiques amenant David à mieux conduire l'étude et à différencier plus facilement les tâches et les savoirs introduits au cours des leçons ${ }^{8}$.

30 Ainsi, l'analyse mésodidactique révèle que, tout au long de la séquence d'enseignement, David tente de préserver la dynamique d'apprentissage en conciliant la différenciation de l'étude et la gestion des groupes. Sur le plan mésogénétique, l'agencement des dispositifs repose sur l'introduction d'objets de savoirs différenciés en fonction du niveau des élèves (voir Tableau 1) et de différents formats pédagogiques et communicationnels (voir Tableau 2). Dans les deux sections suivantes, nous prolongerons cette analyse mésodidactique par une analyse microdidactique prenant appui sur deux évènements remarquables.

\subsection{L'introduction d'un objet matériel support de différenciation}

31 Le premier évènement remarquable retenu illustre une modalité de différenciation des apprentissages mise à l'étude par David en leçon 2. Il concerne une tâche de continuité ${ }^{9}$ au cours de laquelle les élèves doivent effectuer un maximum d'échanges, en coup droit ou en revers. Bien que les contraintes de la tâche soient déjà différenciées (voir Tableau 1), David introduit un objet matériel à destination des joueurs en difficulté une balle au diamètre plus important - leur permettant de travailler dans des conditions de pratique plus confortables et sécuritaires :

«Les élèves peuvent mieux lire les trajectoires et toucher la balle plus facilement.

Ils sont tout de suite placés dans des conditions plus facilitantes » (entretien post

séance 2).

Selon David, la balle est une ressource pédagogique susceptible d'engendrer des transformations importantes chez les élèves en termes de placement et de trajets moteurs. En effet, la taille des balles utilisées a une incidence sur les trajectoires produites et sur les possibilités de relance des élèves. Plus la taille est importante, plus le jeu est ralenti et plus les joueurs ont des facilités pour construire un timing de frappe adéquat. Ils peuvent ainsi se placer plus facilement et développer des repères moteurs plus fins pour accompagner la balle lors de l'exécution du geste. Sur ce point, l'interprétation de l'enseignant converge avec l'analyse a priori selon laquelle l'utilisation d'une balle de 55 millimètres de diamètre (au lieu de $40 \mathrm{~mm}$ ) confère aux joueurs davantage de repères visuels et sensorimoteurs participant à une meilleure lecture des trajectoires et favorisant le toucher. Effectivement, l'analyse a posteriori montre qu'au début de la situation et avec une balle standard $(40 \mathrm{~mm})$, les élèves les 
plus en difficulté, raides sur leurs jambes et explosifs dans leurs frappes, rataient fréquemment la balle et/ou décentraient leurs coups ce qui les empêchaient de réaliser plus de deux échanges. À l'inverse, les élèves en réussite étaient capables de jouer en contrôle à une cadence relativement soutenue (cela se traduit par une augmentation de la vitesse de balle et une horizontalisation des trajectoires). La reprise de la même tâche avec une balle proéminente a entraîné une amélioration de la continuité de l'échange chez les élèves en difficulté, permise par la lenteur du jeu doublement combinée à la grosseur de la balle et à l'accompagnement du geste des pongistes lors de la frappe. En ce sens, la mésogénèse s'enrichit de cet aménagement en favorisant le développement des rétroactions avec le milieu. Au cours de cet évènement, David supervise le travail des élèves et n'intervient que ponctuellement pour réguler leur activité sur des composantes techniques liées principalement à la tenue de raquette et à l'organisation des appuis par rapport à la balle. Sur le plan topogénétique, David adopte une posture d'accompagnement ciblé qui contribue à renforcer la construction des savoirs visés.

En outre, l'analyse a posteriori montre qu'en permettant aux élèves en difficulté de construire la continuité de l'échange, David souhaite limiter la manifestation de comportements déviants. Il considère la mise en activité - et plus spécifiquement l'accès à une réussite immédiate - comme un moyen de lutter contre les ruptures de contrats didactiques, comme en témoigne l'extrait suivant :

«Savoir renvoyer la balle c'est très important, car c'est une condition pour jouer.

Or, si les élèves sentent qu'ils n'arrivent pas à jouer, ils se lassent et décrochent très

vite » (entretien post séance 2).

Ainsi, les remaniements opérés par l'enseignant répondent à deux préoccupations liées au guidage des apprentissages et au contrôle de la classe, s'alimentant l'une l'autre. David propose des modalités d'enseignement différenciées qui permettent aux élèves en difficulté de travailler dans un milieu didactique plus stable et moins incertain. De ce fait, les savoirs techniques qui président à la construction du jeu sont maintenus, mais déclinés différentiellement en fonction du niveau des élèves. Aussi, en proposant des contenus de savoir adaptés aux caractéristiques motrices des élèves, David souhaite favoriser l'engagement et le progrès de tous, « forts » comme « faibles ».

\subsection{La structure de communication au service de la définition des tâches et de la différenciation}

34 L'évènement sélectionné s'inscrit dans une tâche d'opposition aménagée ayant pour objectif d'amener les élèves à exploiter des opportunités d'attaque pour rompre l'échange. Cette tâche est proposée par l'enseignant lors de la troisième leçon et vise à différencier les modalités d'opposition en fonction du niveau des joueurs. En observant les trois instances - David, le collectif de classe, et les deux élèves interrogés (Céline et Bobby respectivement considérés comme des élèves en difficulté et en réussite) -, nous cherchons à comprendre en quoi ce modèle de définition trilogique offre des perspectives de différenciation effectives. Comme indiqué ci-dessous, cette tâche d'opposition aménagée est différenciée de sorte que les élèves en difficulté (qui poursuivent des enjeux de savoir relatifs au placement de balle) doivent viser des zones latérales pour marquer un point bonifié à la suite d'un banco ${ }^{10}$. Les élèves en réussite doivent quant à eux réaliser un smatch, à la suite d'une annonce, pour que le point soit bonifié en cas de succès (voir Tableau 3). 
Tableau 3 : Extrait de la leçon 3 : verbatim des interactions en classe.

Tableau 3: An excerpt from lesson 3: verbatim of classroom interactions

David [installe les élèves dans les tribunes et se tient face à eux] : "On poursuit avec la situation du banco sauf que cette fois-ci on va modifier légèrement les consignes. Tout le monde a bien compris le principe du banco déjà »?

La classe : " oui »

David : "Pour les élèves qui sont sur l'atelier placement de balle comme Céline. Si vous annoncez la zone "rouge ou bleue " [il désigne les tables sur lesquelles sont installées des cibles rouges et bleues placées respectivement à droite et à gauche de la table] lorsque vous pensez être en situation de marquer le point et que vous réussissez effectivement à marquer le point derrière vous gagnez un bonus de 10 points. Rappellenous combien de «banco » par point Céline »?

Céline : "On a le droit d'en faire un seul»

David : "Très bien. Et combien de «Banco » par match au total »?

Céline : "On peut en faire 3 ».

David : "Oui exact. Du coup, ceux de l'atelier "smatch », même consigne sauf que vous vous n'avez pas de zone. Vous voyez? [Il désigne les tables qui ne comportent pas de zones rouges ou bleues]. Pour vous 10 points bonus si vous annoncez «banco » avant le smatch. Si l'adversaire renvoie le smatch il marque 5 points et le jeu s'arrête. Bobby, tu nous rappelles ce qu'est un smatch »?

Bobby : "C'est quand on tape fort dans la balle »

David : "Et quand est ce que je peux taper fort dans la balle »?

Bobby : "Quand la balle de l'adversaire est en cloche »

David : "C'est quoi l'autre condition. [Il attend quelques secondes alors que d'autres élèves lèvent la main et commencent à donner oralement des réponses sans avoir été préalablement invités à le faire] Par rapport au placement »?

Bobby : "Ah oui, il faut être proche de la table »

L'analyse microdidactique de cet extrait montre que David introduit la notion de jeu placé ou accéléré en fonction des profils de joueurs. Dans la continuité de la tâche précédente, l'enseignant oriente les élèves vers un enjeu de savoir lié à la reconnaissance d'une situation favorable de marque (voir Tableau 1). Pour David :

« au niveau 1 de compétence attendue il faut que les élèves comprennent ce que ça veut dire être en situation favorable. Pour ça je leur donne des repères simples par rapport à la trajectoire de balle et au placement du joueur » (entretien post leçon 3).

Dans l'extrait rapporté ci-dessus, le recours à une organisation trilogique permet à l'enseignant de définir un enjeu de savoir distinct pour chacun des profils (fort et faible). Céline et Bobby sont donc considérés comme une instance élève participant momentanément à l'explicitation de la tâche et à la construction d'une référence différentielle. Les élèves les plus proches des attendus de la compétence s'orientent vers la rupture par l'accélération tandis que les élèves les plus éloignés des exigences programmatiques s'orientent vers le placement de balle.

D'un point de vue topogénétique, David adopte volontairement une position basse en laissant aux élèves le soin de formaliser certains éléments de connaissance. En ce sens, il opte pour un discours questionnant permettant aux élèves désignés, Céline et Bobby, de rebondir sur les remarques et les questions posées lors de la phase de définition. Sur le plan chronogénétique, David introduit de nouvelles consignes qui permettent de faire évoluer différentiellement la situation du banco. Il pointe les savoirs communs aux deux types de tâches et met en avant les contenus spécifiques abordés au cours de cette situation. 
$37 \mathrm{Au}$ cours de la situation, David régule l'activité des élèves de l'atelier «smatch » en référence à leurs choix et non en fonction de leur placement corporel. Il apporte donc aux élèves des informations concernant les savoirs tactiques qui interrogent en arrièreplan la construction d'un projet de jeu basé sur les principes de rupture de l'échange. À l'inverse, l'enseignant corrige la posture des élèves de l'atelier "placement de balle » en les reprenant fréquemment sur leur prise de raquette et sur l'accompagnement de la balle en revers. Dans cette optique, il valorise des savoirs techniques, en partie encapsulés dans des gestes de type démonstratifs, et ayant pour objectif d'étoffer le bagage moteur des joueurs. Ainsi, l'analyse a posteriori montre que les gestes de régulation de David marquent un positionnement différencié (en termes de modalité et en termes de contenus d'enseignement) dans l'accompagnement et le suivi de l'activité des élèves. Il réalise des interventions distinctes en référence au niveau de pratique des joueurs.

\section{Discussion conclusive}

\subsection{Des dilemmes professionnels réactualisés}

Les résultats pointent certaines incohérences telles que la mise à l'étude de contenus différenciés selon un découpage arbitraire de la compétence attendue ou encore le manque de visibilité des savoirs, imputables au format de communication adopté (organisation magistrale). Pour revenir sur le premier point, l'analyse des EAC montre qu'en prenant appui sur les programmes EPS de collège l'enseignant choisit d'orienter volontairement les élèves « en difficulté » vers le placement de balle et les élèves «en réussite » vers l'accélération (entretien post séance 3). Dans cette logique, il élabore plusieurs types de tâches et introduit des contenus d'enseignement différenciés en fonction du profil des élèves. En faisant cela, David choisit à la place des joueurs et les cantonne à un registre potentiellement réducteur et inadapté à leurs besoins. Concrètement, les élèves en difficulté risquent d'être enfermés dans une culture de pratique qu'ils auront du mal à délaisser tandis que les élèves en réussite auront peutêtre plus de facilité pour développer leur répertoire technico-tactique. En ce sens, l'interprétation que l'enseignant fait de la compétence attendue, sous couvert d'intentions didactiques parfaitement légitimes (différencier les savoirs et les contenus afin de permettre à tous les élèves d'apprendre à rompre l'échange), l'incite à proposer des formats d'apprentissages hermétiques et injonctifs, pouvant contribuer à maintenir des différences entre les joueurs.

Au-delà, les résultats soulignent que l'enseignant débutant exerçant en milieu difficile se heurte à des dilemmes professionnels réactualisés au jour le jour. L'activité de l'enseignant est tiraillée entre d'un côté, la nécessité de faire progresser tous les élèves au cours de la séquence, et de l'autre, la difficulté à gérer leur engagement dans les tâches. Cette tension peut occasionner des frustrations et influencer significativement les manières dont l'enseignant différencie l'étude tout en aménageant des conditions propices au travail. Sur ce point, nos analyses enrichissent, de façon complémentaire, les résultats mis au jour par les recherches inhérentes au milieu difficile (Girard \& Vors, 2018 ; Petiot et Visioli, 2017 ; Vors \& Gal-Petitfaux, 2008, 2009, 2011, 2015 ; Vors, GalPetitfaux, \& Potdevin, 2015) notamment à propos des stratégies visant à maintenir l'ordre au sein de la classe. En effet, l'étude montre que la différenciation des tâches et 
des enjeux de savoir (voir tableau 1) contribue à instaurer un cadre de travail structurant pour les élèves, pouvant ainsi limiter l'apparition de comportements déviants tout comme les stratégies de masquage/ostension (Vors \& GalPetitfaux, 2009).

En outre, la mise en place de structures de communication non exclusivement dilogiques ou magistrales (voir extrait de corpus présenté dans le tableau 3) met en lumière un haut niveau d'articulation entre les préoccupations organisationnelles et didactiques de l'enseignant, confirmant ainsi certains travaux menés en didactique de l'EPS (Brière-Guenoun, 2015; Monnier \& Amade-Escot, 2009; Poggi, 2014). D'une certaine façon, si l'adossement de contenus d'enseignement différenciés aux dispositifs organisationnels contribue à assurer la gestion collective et individuelle de la classe (Petiot \& Visioli, 2017), il témoigne aussi de leur enracinement, parfois tacite, dans des pratiques différentielles, voire inégalitaires vis-à-vis de l'appropriation des savoirs (Brière-Guenoun, Couchot-Schiex, Poggi, \& Verscheure, 2018). Comme l'ont montré les résultats, le recours à une organisation trilogique permet notamment à l'enseignant d'expliciter et de différencier les enjeux de savoirs de la situation pour optimiser les temps de régulation in situ. En EPS, les stratégies de transmission des consignes sont spécifiques au regard des espaces ouverts et non hermétiques où elles sont diffusées : le bruit étant une composante essentielle de l'activité, les choix liés à l'aménagement des transitions entre les phases de définition et les phases de mise en pratique influencent fortement la dynamique de travail du groupe classe ainsi que la densité des régulations didactiques opérées par l'enseignant au cours de la situation. Ce choix organisationnel rend compte d'une forme d'économie didactique dont le but est de faciliter l'engagement des élèves dans le travail tout en permettant aux acteurs de se focaliser sur les processus de l'étude et l'acquisition des savoirs. Mais il tend simultanément à renforcer la mise à l'étude différentielle des savoirs (tactiques versus techniques) selon le niveau de pratique des élèves.

41 Finalement, si la recherche présentée permet de dévoiler les modalités de différenciation en milieu difficile, la spécificité des résultats ne doit pas évacuer leur portée générique. Considérant le milieu difficile comme "un miroir amplifiant » du fonctionnement (ou des dysfonctionnements) de l'ensemble des systèmes pédagogiques, l'analyse des gestes de différenciation de l'enseignant peut à cet égard ouvrir des perspectives de compréhension dans d'autres contextes d'enseignement.

\subsection{Interroger les perspectives transformatives des pratiques de différenciation}

Nos analyses montrent notamment que l'influence des prescriptions institutionnelles pèse fortement sur l'activité de l'enseignant. Désireux de suivre les recommandations des programmes, ce dernier organise la séquence d'enseignement en référence à la compétence attendue de niveau 1 . Ce constat rejoint les travaux menés en ergonomie qui mettent en évidence un double mouvement des prescriptions (Daniellou, 2002 ; Saujat, 2010 ; Six, 1999). Ces auteurs distinguent :

a. les prescriptions descendantes provenant d'une structure organisationnelle qui transmet des directives claires concernant les objectifs de travail visés (les injonctions institutionnelles) ; 
b. les prescriptions ascendantes ou remontantes qui émergent du contexte de travail et/ou de la matière travaillée (ici l'activité des élèves par exemple).

Elles peuvent alors entrer en conflit, voire s'opposer, aux prescriptions hiérarchiques et amènent le professionnel à effectuer des choix situationnels. Les résultats montrent également que l'enseignant redéfinit les modalités de mise en œuvre des prescriptions selon l'avancée du temps didactique, selon le niveau des élèves et selon des critères de faisabilité. De ce fait, il concilie des préoccupations liées aux contenus à enseigner (de type technique et tactique), à la gestion de classe (formats de travail, aménagement de l'espace et structure de communication) et à sa zone de confort dans l'exercice du métier. Ainsi, cette étude révèle l'ingéniosité des acteurs dans leur capacité à reconfigurer les prescriptions pour les adapter à leur contexte professionnel et à leurs buts subjectifs en lien avec leur construction du métier (Brière-Guenoun, 2017). Si ce corolaire va dans le sens des résultats produits en clinique de l'activité sur les compromis entre les enjeux épistémiques, la gestion de la classe et la préservation de soi (Saujat, 2010), il met également en lumière, et de façon originale, la manière avec laquelle cet enseignant débutant se propose de mettre en œuvre les différents niveaux de prescription au sein de la classe. Bien que cette recherche ne nous donne pas d'indications sur ce point, nous pouvons supposer que les dispositifs de formation initiale dans le cursus des enseignants d'EPS, confrontant très tôt les étudiants à la réalité du milieu professionnel par l'intermédiaire de stage de formation, permettent à ces derniers d'intérioriser peu à peu certains gestes professionnels faisant souvent défaut aux enseignants débutants. Si cette hypothèse n'est pas directement vérifiable, elle pourrait faire l'objet d'une analyse comparative ultérieure visant à éclairer les procès de construction de la professionnalité au sein de la corporation des enseignants d'EPS.

Par ailleurs, la mobilisation d'un cadre ergonomique, initialement destiné à traiter des questions relatives au développement professionnel, interroge au-delà les perspectives transformatives $\mathrm{du}$ dispositif de recherche présenté. Les entretiens d'autoconfrontation semblent influencer le regard et les prospectives de l'enseignant qui est amené à revivre des épisodes de son activité professionnelle. En particulier le choix des évènements remarquables, qui s'établit en lien direct avec nos préoccupations initiales de recherche, oriente singulièrement les axes de l'analyse à laquelle est invité l'enseignant en reconceptualisant sa propre activité. De fait, le cadre d'observation, induit par la recherche, peut amener l'enseignant à se recentrer de façon plus fine (et peut-être aussi plus artificielle) sur les instances impersonnelles du métier (et notamment les prescriptions institutionnelles qui entérinent la conformité aux orientations des programmes). Le recours aux entretiens d'autoconfrontation peut mettre au jour des conflits de préoccupations sous-jacents à l'activité de l'enseignant et articulés aux diverses instances du métier définies par Clot (2008) et Kotulski et al. (2011). Leur articulation avec un dispositif de type didactique, soutenu par le choix des évènements remarquables, révèle l'emboitement des dimensions subjectives, didactiques et institutionnelles inscrites dans les gestes de différenciation de l'enseignant (Brière-Guenoun, 2017).

Si les visées transformatives ne sont pas initialement inscrites dans le dispositif de recherche, il semblerait tout de même, à la lumière des résultats pointés au cours de cette étude, que certaines modalités de recherche-intervention soient plus efficaces que d'autres pour asseoir une vigilance didactique minimale au sein de la classe et 
repositionner la question des savoirs au cœur du processus d'enseignement. Les travaux actuels concernant les "interventions - recherches" (Espinassy, BrièreGuenoun, \& Félix, 2018), les démarches collaboratives de co-conception (Cèbe \& Goigoux, 2018) ou les dispositifs de formation (Butlen \& Masselot, 2018) s'accordent d'ailleurs sur la nécessité de prendre en compte les pratiques réelles pour développer avec les acteurs de nouveaux outils d'enseignement articulés aux enjeux didactiques.

\subsection{Les limites soulevées : entre études de cas et généralisation}

Inscrit dans une approche didactique et clinique visant à questionner la réalité des pratiques d'enseignement, notre dispositif méthodologique se restreint à des études de cas donnant à voir, sous un éclairage particulier, les conditions d'apprentissage et de différenciation installées par un enseignant débutant exerçant en milieu difficile. En conséquence, il se démarque de certaines recherches quantitatives en sciences sociales qui appréhendent des modalités d'investigation bien plus larges en cherchant à toucher un échantillonnage conséquent pour tester la validité des hypothèses de recherche. En outre, il s'appuie sur une construction expérimentale légère (voir la méthode) ne permettant pas de contenir ou d'isoler certaines variables d'ordre didactique et contextuel notamment. Dans cette perspective, notre travail propose modestement de reconstruire, par le biais d'une analyse spécifique et ascendante, des modèles de portée génériques pouvant trouver une certaine résonnance dans d'autres contextes.

Si nous considérons, à l'instar de l'approche comparatiste, que le travail solidaire des aspects génériques et spécifiques renforce l'intelligibilité des processus didactiques (Amade-Escot, 2007), nous pensons tout de même que les résultats de notre étude méritent d'être observés à la lumière des dispositions singulières au sein desquelles ils ont été recueillis. Aussi, "la question des modes de construction des savoirs et corrélativement celle de la capacité d'engendrer des assertions de portée générale » mérite d'être pensée de façon raisonnée et soumise à un vif débat épistémologique (Fabiani, 2007, p. 10). Sur ce point, Latour (2005) nous met en garde contre « une routine scolaire qui consisterait systématiquement à accoupler une étude de cas à une visée généralisante » (p. 184).

\section{BIBLIOGRAPHIE}

Amade-Escot, C. (2007). Le didactique. Paris : Éditions revue EPS.

Amade-Escot, C., \& Venturini, P. (2009). Le milieu didactique : d'une étude empirique en contexte difficile à une réflexion sur le concept. Éducation \& Didactique, 3(1), 7-43.

Baluteau, F. (2014). La différenciation pédagogique : quels modes d'explication sociologique?

Revue française de pédagogie, 188, 51-62. 
Bocchi, P-C. (2012). Les formes de régulation de l'activité des élèves. Recherche en éducation, HorsSérie, 4, 55-67.

Brière-Guenoun, F. (2015). Les gestes du professeur d'éducation physique en milieu difficile : de multiples compromis au service de la co-construction des savoirs. Carrefours de l'éducation, 40(2), 85-103.

Brière-Guenoun, F. (2017). Instruire les gestes didactiques de métier. Quelles perspectives pour la formation des enseignants? Presses Universitaires de Rennes.

Brière-Guenoun, F., \& Poggi, M-P. (2015). L'agir enseignant à l'interface du sociologique et du didactique. eJRIEPS, 34 .

Brière-Guenoun, F., Couchot-Schiex, S., Poggi, M-P., \& Verscheure, I. (2018). Les inégalités d'accès aux savoirs se construisent aussi en éducation physique et sportive... Analyses didactiques et sociologiques. PUFC.

Bruno, F., Félix, C., \& Saujat, F. (2015). Les programmes de prévention et de lutte contre le décrochage scolaire et leurs conséquences sur le travail enseignant : revue de littérature. Revue Française de Pédagogie, 193, 89-104.

Butlen, D., Mangiante-Orsola, C., \& Masselot, P. (2017). Routines et gestes professionnels, un outil pour l'analyse des pratiques effectives et pour la formation des pratiques des professeurs des écoles en mathématiques. Recherches en didactiques, 24(2), 25-40.

Butlen, D., \& Masselot, P. (2018). De la recherche à la formation : enrichir les pratiques des enseignants pour favoriser les apprentissages des élèves en mathématiques. Recherche \& formation, 87(1), 61-75.

Cèbe, S., \& Goigoux, R. (2018). Lutter contre les inégalités : outiller pour former les enseignants. Recherche \& formation, 87(1), 77-96.

Charles-Pezard, M., Butlen, D., \& Masselot, P. (2012). Professeurs des écoles débutants en ZEP. Quelles pratiques? Quelle formation? Recherche et Formation, 71, 133-134.

Chesne, J-F., Do, C-L., Jego, S., Briant, P., Lefresne, F., \& Simonis-Sueur, C. (2014). Enquête TALIS (2013). Enseignant en France : un métier solitaire ? Note d'information $n^{\circ} 23$, Depp-MENESR.

Clot, Y. (1999). La fonction psychologique du travail. Paris : PUF, Le travail humain.

Clot, Y. (2008). Travail et pouvoir d'agir. Paris : PUF.

Conseil National d'Évaluation du Système Scolaire. (2016). Inégalités sociales et migratoires. Comment l'école amplifie-t-elle les inégalités. Rapport scientifique.

Crahay, M. (2003). Peut-on lutter contre l'échec scolaire ? Bruxelles : De Boeck.

Crahay, M., \& Wanlin, P. (2012). La pensée des enseignants pendant l'interaction en classe. Une revue de littérature anglophone. Éducation et didactique, 6(1), 9-46.

Daniellou, F. (2002). Le travail des prescriptions. In Actes du 37e Congrès de la SELF « Nouvelles formes de travail, nouvelles formes d'analyse ", Aix en Provence, pp. 9-16.

Elbaz, F. (1981). The teacher's « practical knowledge »: Report of a case study. Curriculum Inquiry, 11, 43-71.

Elbaz, F. (1983). Teacher thinking: A study of practical knowledge. London : Croon Heln. 
Espinassy, L., Brière-Guenoun, F., \& Félix, C. (2018). De l'intervention-recherche dans les dispositifs de lutte contre les inégalités scolaires à la formation des enseignants. Recherche \& formation, 87(1), 47-60.

Fabiani, J-L. (2007). La généralisation dans les sciences historiques. Obstacle épistémologique ou ambition légitime? Annales. Histoire, Sciences Sociales 2007/1 (62e année), 9-28.

Gal-Petitfaux, N., \& Saujat, F. (2018). Les gestes professionnels de l'enseignant. In M. Travert \& O. Rey (Eds.), L'engagement de l'élève en EPS. D'une approche pluridisciplinaire aux perspectives professionnelles, Dossier EPS n ${ }^{\circ}$ 85, édition Revue EPS.

Gal-Petitfaux, N., Vors, O., \& Cizeron, M. (2011). Interactions enseignant-élèves et perception des transgressions en classe d'Éducation physique dans les collèges difficiles en France. In Mieux former pour agir dans une société en mouvement (pp. 20-26). Presses de l'Université du Québec.

Girard, A., \& Vors, O. (2018). L'élève « difficile ». In M. Travert \& O. Rey, L'engagement de l'élève en EPS. D'une approche pluridisciplinaire aux perspectives professionnelles, Dossier EPS $n^{\circ} 85$, édition Revue EPS.

Kherroubi, M., \& Rochex, J-Y. (2004). La recherche en éducation et les ZEP en France. Apprentissages et exercice professionnel en ZEP : résultats, analyses, interprétations. Revue française de pédagogie, 146, 115-190.

Kirouac, M-J. (2010). L'intégration et la mise en œuvre de la pratique de différenciation pédagogique chez les enseignant.es québécois du premier cycle du secondaire. Mémoire en sciences de l'éducation. Montréal : Université de Montréal.

Kostulski, K., Clot, Y., Litim, M., \& Plateau, S. (2011). L'horizon incertain de la transformation en clinique de l'activité : une intervention dans le champ de l'éducation surveillée. Activités, 8(1).

Latour, B. (2005). Reassembling the social. An introduction to actor-network theory. Oxford : Oxford University Press.

Legrand, L. (1986). La différenciation de la pédagogie. Paris : PUF.

Leutenegger, F. (2000). Construction d'une « clinique » pour le didactique. Une étude des phénomènes temporels de l'enseignement. Recherche en Didactique des Mathématiques, 20/2, 209-250.

Leutenegger, F. (2008). L'entrée dans un code écrit à l'école enfantine et l'articulation entre le collectif et l'individuel : comparaison de deux études de cas. Éducation \& didactique, 2(2), 7-42.

Leutenegger, F. (2009). Le temps d'instruire. Approche clinique et expérimentale du didactique ordinaire en mathématiques. Bruxelles : Peter Lang.

Ligozat, F. (2008) Un point de vue de didactique comparée sur la classe de mathématiques. Étude de l'action conjointe du professeur et des élèves à propos de l'enseignement / apprentissage de la mesure des grandeurs dans des classes françaises et suisse romandes. Thèse de doctorat en Sciences de l'Éducation, Université de Genève et Université de Provence.

Meirieu, P. (1987). Apprendre... oui, mais comment ? Paris : ESF.

Meirieu, P. (2013). Pédagogie : des lieux communs aux concepts clés, Paris : ESF.

Mercier, A., Schubauer-Leoni, M-L., \& Sensevy, G. (2002). Vers une didactique comparée. Revue Française de pédagogie, 141, 5-16.

Monfroy, B. (2002). La définition des élèves en difficulté en ZEP : le discours des enseignants de l'école primaire. Revue française de pédagogie, 140, 33-40. 
Monnier, N., \& Amade-Escot, C. (2009). L'activité didactique empêchée : outil d'intelligibilité de la pratique enseignante en milieu difficile. Revue française de pédagogie, 168, 59-73.

Petiot, O., \& Visioli, J. (2017). Le dilemme entre la gestion collective et la gestion individuelle de la classe par un enseignant d'EPS expert exerçant en milieu difficile : une étude exploratoire. Movement and sport sciences, 95, 65-79.

Poggi, M-P. (2014). Enseigner l'EPS en milieu difficile : vers une confrontation en actes de différents niveaux de contexte dans le cadre d'une approche socio-didactique. Questions Vives, 22.

Prud'homme, L., Dolbec, A., Brodeur, M., Presseau, A., \& Martineau, S. (2005). La construction d'un îlot de rationalité autour du concept de différenciation pédagogique. Journal of the Canadian Association for Curriculum Studies, 3, 1-31.

Rayou, P., \& Sensevy, G. (2014). Contrat didactique et contextes sociaux. La structure d'arrièreplan des apprentissages. Revue française de pédagogie, 188, 23-38.

Ria, L. (2009). De l'analyse de l'activité des enseignants débutants en milieu difficile à la conception de dispositifs de formation. In M. Durand \& L. Filliettaz (Eds.), La place du travail dans la formation des adultes (pp. 217-243). Paris : PUF.

Ria, L., Saury, J., Sève, C., \& Durand, M. (2001). Les dilemmes des enseignants débutants. Études lors des premières expériences de classe en Éducation physique. Science et motricité, 42, 47-58.

Rochex, J-Y. (2016). Faut-il crier haro sur l'éducation prioritaire ? Analyses et controverses sur une politique incertaine. Revue française de pédagogie, 194(1), 91-108.

Rochex, J-Y., \& Crinon, J. (2011). La construction des inégalités scolaires. Au cœur des pratiques et des dispositifs d'enseignement. Rennes : PUR.

Rouve, M., \& Ria, L. (2008). Analyse de l'activité professionnelle d'enseignants néo-titulaires en réseau ambition réussite : études de cas. Travail \& Formation en Éducation, 1.

Saujat, F. (2010). Travail, formation et développement des professionnels de l'éducation : voies de recherche en sciences de l'éducation, Note de synthèse pour l'Habilitation à Diriger des Recherches, Université de Provence (Aix-Marseille I).

Schubauer-Leoni, M-L. (1986). Le contrat didactique : un cadre interprétatif pour comprendre les savoirs manifestés par les élèves en mathématiques. Journal Européen de Psychologie de l'Éducation, 1(2), 139-153.

Schubauer-Leoni, M-L (1996). Étude du contrat didactique pour des élèves en difficulté en mathématiques. Problématique didactique et/ou psychosociale. In C. Raisky \& M. Caillot (Eds.), Au-delà des didactiques, le didactique, Débats autour de concepts fédérateurs (pp. 159-189). Bruxelles : De Boeck.

Schubauer-Leoni, M-L., \& Leutenegger, F. (2002). Expliquer et comprendre dans une approche clinique/expérimentale du didactique ordinaire. In F. Leutenegger \& M. Saada-Robert (Eds.), Expliquer et comprendre en sciences de l'éducation (pp. 227-251). Paris, Bruxelles : De Boeck.

Sensevy, G., \& Mercier, A. (2007). Agir ensemble. L'action conjointe du professeur et des élèves dans le système didactique. Rennes : PUR.

Six, F. (1999). De la prescription à la préparation du travail : apports de l'ergonomie à la prévention et à l'organisation du travail sur les chantiers du bâtiment. Note de synthèse pour l'Habilitation à Diriger des Recherches, Université Charles de Gaulle (Lille III). 
Toullec-Théry, M. (2016). L'individualisation permet-elle de lutter contre les inégalités sociales dans la classe ? Contribution au rapport du Cnesco : Comment l'école amplifie-t-elle les inégalités sociales et migratoires?

Toullec-Théry, M., \& Marlot, C. (2013). Les déterminations du phénomène de différenciation didactique passive dans les pratiques d'aide ordinaire à l'école élémentaire. Revue française de pédagogie, 182(1), 41-54.

Vors, O., \& Gal-Petitfaux, N. (2008). Mettre une classe au travail en réseau ambition réussite : des formes typiques d'interaction enseignant-élèves lors de leçons d'EPS. Revue travail et formation en éducation, UMR ADEF, P3.

Vors, O., \& Gal-Petitfaux, N. (2009). Construire une activité collective de travail dans une classe d'EPS en « Réseau ambition réussite » : entre masquage et ostentation. eJRIEPS, 156-177.

Vors, O., \& Gal-Petitfaux, N. (2011). Situations de travail par ateliers et configuration de l'activité collective en classe « Réseau Ambition Réussite ». eJRIEPS, 96-115

Vors, O., \& Gal-Petitfaux, N. (2015). Relation between students' involvement and teacher management strategies in French "difficult" classrooms. Physical Education and Sport Pedagogy, 20(6), 647-669.

Vors, O., Gal-Petitfaux, N., \& Potdevin, F. (2015). A successful form of trade-off in compensatory policy classrooms. European Physical Education Review, 21(3), 340-361.

\section{NOTES}

1. Tels que le refus de travailler, le décrochage de la pratique physique, le chahut, l'agitation ou encore l'indolence.

2. D'ailleurs, le rapport du CNESCO (2016) sur l'origine des inégalités sociales et migratoires à l'école, montre qu'au cours de la dernière décennie le nombre d'enseignants débutants ou peu expérimentés (moins de 30 ans) intervenant en éducation prioritaire a progressé de façon importante (21,7 \% en 2008 contre $26,3 \%$ en 2015$)$.

3. Pour un développement, voir Brière-Guenoun (2017).

4. À la suite d'Elbaz $(1981,1983)$, nous pensons que l'expérience est corrélée à l'ancienneté de l'enseignant. Elle distingue très précisément le néophyte ou l'apprenant (aucune expérience), le débutant (moins de cinq années d'expérience) et le chevronné (plus de dix années d'expérience).

5. L'étude présentée s'inscrit dans une recherche comparant l'activité de l'enseignant dans deux cycles d'enseignement (le tennis de table et le demi-fond).

6. En référence aux programmes collège de 2008, l'enseignant doit viser le niveau 1 de compétence attendue avec des élèves de $4^{\mathrm{e}}$ débutants dans l'activité (zéro heure de pratique effective antérieure). Notons toutefois que depuis, les enseignants sont soumis à de nouveaux programmes EPS qui leur offrent davantage de liberté pédagogique concernant le découpage institutionnel du temps scolaire.

7. Elle consiste à observer les conduites motrices des élèves pour identifier leurs besoins en termes d'acquisition (ici acquisitions techniques et tactiques).

8. Nous étofferons ce point lors de la deuxième analyse microdidactique.

9. Celle-ci a pour objectif d'amener les élèves à effectuer un maximum d'échanges. David différencie les contraintes de la tâche en demandant aux joueurs "forts » d'effectuer des échanges en alternant coup droit et revers, aux joueurs "faibles» de réaliser des échanges à partir d'un coup préférentiel (coup droit ou revers), tandis qu'il ne donne aucune contrainte aux joueurs en grande difficulté (jeu libre). 
10. Les joueurs doivent annoncer « banco » lorsqu'ils pensent être en mesure de marquer le point derrière la frappe. Chaque joueur a le droit d'utiliser 3 « banco » par match.

\section{RÉSUMÉS}

Cette recherche s'intéresse aux gestes de différenciation d'un enseignant d'éducation physique et sportive (EPS) débutant exerçant en éducation prioritaire. Elle s'inscrit dans une approche comparatiste en didactique, enrichie par les fondements théoriques et méthodologiques de la clinique de l'activité. Nous cherchons à comprendre comment un enseignant d'EPS débutant peut concilier les diverses contraintes qui pèsent sur son activité, liées notamment aux caractéristiques du contexte, à ses motifs d'agir et aux prescriptions institutionnelles, pour conduire et différencier son enseignement. Les résultats montrent que l'instauration de conditions propices au travail est permise grâce à un aménagement souple du milieu didactique et à la construction de diverses structures de communication centrées sur les apprentissages. Les gestes de différenciation résultent de multiples dilemmes tels que gérer la classe, conduire l'enseignement pour tous et chacun, favoriser des savoirs de type tactique ou technique et respecter/adapter les prescriptions. Au-delà, sont discutées des perspectives de développement du pouvoir d'agir des enseignants débutants exerçant en milieu difficile.

This research focuses on the differentiation of a beginner physical education and sports teacher (PSE) exercising in priority education. It is part of a comparative approach to didactics, enriched by the theoretical and methodological foundations of the clinic ofactivity. We seek to understand how beginner teachers can reconcile the various constraints on their activity, particularly those relating to the characteristics of the context, to their motives for action and institutional requirements, to lead and differentiate their teaching. The results show that the creation of conditions conducive to work is made possible through flexible management of the didactic environment and the construction of various communication structures centered on learning. Differentiation results from multiple dilemmas such as managing the class, conducting lessons for the benefit of each and every student, promoting tactical or technical knowledge and respecting/adapting prescriptions. Beyond this, we discuss perspectives for developing the power to act of beginner teachers exercising in a difficult environment.

\section{INDEX}

Mots-clés : activité, didactique comparée, éducation physique et sportive, gestes de différenciation, structures de communication

Keywords : activity, comparative didactics, physical education and sports, differentiation, structures of communication 


\section{AUTEURS}

\section{TEDDY MAYEKO}

ADEF, EA 4671, Aix-Marseille Université, 27 avenue Magellan, Créteil, 94000,

tdy.mayeko@gmail.com

\section{FABIENNE BRIÈRE-GUENOUN}

ADEF, EA 4671, Aix-Marseille Université, 2 avenue Jules Isaac, 13100, Aix-en-Provence, briere.f@wanadoo.fr 